\title{
On a linear differential game of optimal approach of many pursuers with one evader
}

\begin{abstract}
We consider a differential game of approach of many pursuers and one evader. The motions of all players are described by linear systems of the same type. Control functions of players are subject to integral constraints. The duration of the game is fixed. The payoff functional of the differential game is the minimum of the distances between the evader and the pursuers when the game terminates. The pursuers try to minimize the payoff functional, and the evader tries to maximize it. We obtain estimates for the payoff functional of the game, which can be guaranteed by players and explicitly describe the strategies of the players. From here we obtain that in some specific cases the value of the game exists, and optimal strategies can be constructed. An illustrative example is considered.
\end{abstract}

Keyword: Control; Evader; Payoff functional; Programmed maximin; Pursuer; Strategy 